\title{
The effects of grain structure on electromigration failure of the lead-free solder bump
}

\author{
Yuanxiang Zhang(1), Lihua Liang(2), Dongdong Zhu(1) \\ (1) Key Laboratory of Air-driven Equipment Technology of Zhejiang Province, Quzhou University, Quzhou 324000, CHINA \\ e-mail: zhangyx@qzu.zj.cn \\ (2) College of Mechanical Engineering, Zhejiang University of Technology, Hangzhou 310014, CHINA
}

\section{SUMMARY}

This paper carries out an electromigration (EM) acceleration test on ball grid array (BGA) samples with Sn96.5/Ag3.0/Cu0.5 solder bumps under constant temperature, and characterizes the structure of $\beta$-Sn grains in the lead-free solder bumps. The EM failure modes of the solder bumps of different grain structures were analysed, aiming to disclose the effect of grain structure on the EM failure. Considering the driving forces of the EM (i.e. electron wind force, stress gradient, temperature gradient and atomic density gradient), the atomic density integral (ADI) method was introduced to simulate the void formation and failure lifetime of the EM. The simulation results show that solder bump reliability and failure mode are greatly affected by grain orientation, in that the EM failure occurs rapidly when the c-axis of grain structure of the solder bump is strongly misaligned, or almost perpendicular, to the current direction. The double grain solder bump with grain boundary parallel to current direction boasts a small EM failure and thus a long lifetime.

KEY WORDS: electromigration (EM); solder bump; grain orientation; finite-element modelling (FEM).

\section{INTRODUCTION}

Assembly and packaging is the final manufacturing process transforming semiconductor devices into functional products for end users. Packaging technology is developing simultaneously with semiconductor device technologies, which can provide electrical connection for signal and power, thermal dissipation paths, and mechanical protection. Usually, solder bumps are the weakest part in electronic package, which are subjected to a combination of electrical, thermal, and mechanical loads. As electronics industry continues to push for high performance and the miniaturization of electronic devices, the increased current density can lead to electromigration failure in solder bumps of electronic package. Electromigration is a mass diffusion process phenomenon which happens as a result of an exchange of momentum between conducting electrons and diffusing metal atoms of solder 
bump. As a result, material is depleted from the cathode end and accumulates at the anode end, which, over time, results in an increase in the resistance and may ultimately lead to failure of the devices [1-3]. The increase of the operating current in interconnects inevitably causes the current density to be carried by each solder bump to rise dramatically. Therefore, electromigration has become critical reliability issue for solder bumps in electronic package.

Traditionally, lead-tin (Pb-Sn) alloys have been widely used as solder alloys in microelectronics. Due to the potential hazards associated with the toxicity of $P b, P b-S n$ solder bumps have been replaced by $\mathrm{Sn}$-based solder bumps. In semiconductor industry, $\mathrm{Sn}-\mathrm{Ag}-\mathrm{Cu}$ is the most common solder alloy for $\mathrm{Sn}$-based solder joints. Typical $\mathrm{SnAgCu}$ alloys contain over 95 $w t$. \% $S n$ and are usually comprised of one or a few large $\beta$-Sn grains after solidification. $\beta$-Sn has a body-centred-tetragonal (BCT) lattice structure, with the lattice constant $a=b=0.583 \mathrm{~nm}$ and $c=0.318 \mathrm{~nm}$. This anisotropic structure can produce different characteristics in relation with its crystallographic orientation, thereby dominating the physical and chemical properties of solder joints [4-6]. Since solders usually form one or a few large grains, the microstructure of the solder plays an important role in the electromigration induced degradation. In particular, the effect of $S n$ grain orientation on electromigration induced failure is a very important issue in flip-chip solder joints [7-8]. Lu et al. [9] proposed two EM degradation modes in high $S n$-based $P b$-free solder joints that are associated with different diffusion process. Mode-I is associated with $S n$ self-diffusion when the $c$-axis is perpendicular or completely misaligned with the current direction, and results in a void formation along the cathode interface. Mode-II occurs as the $S n c$-axis was aligned with the current direction, and results in fast dissolution of under bump metallization (UBM) along the crystal $c$ axis. Lee et al. [10] also reported that the $\beta$-Sn grain orientation played a crucial role in the dissolution rate of the cathode $\mathrm{Cu}$, which resulted in different EM failure life.

The electromigration-induced degradation process is challenging to model because it involves the complex mass diffusion process coupled with the electrical, thermal and mechanical physical phenomena. Lots of effort has been made to develop the computational electromigration models for Sn-based solder joints [11-14]. However, most of the models treat $S n$ grains as isotropic and do not account for the strong anisotropy of $\beta$-Sn grains. In the current literature few models showing the effect of grain structure on the electromigration failure of solder bump, but none of them include the effect of grain anisotropy, [15-16].

In this paper, the electromigration acceleration test was carried out on the $\mathrm{Sn} 96.5 \mathrm{Ag} 3.0 \mathrm{Cu} 0.5$ solder bump to observe the characteristics of the grain structure of the solder bump and study the electromigration failure modes of solder bump with different grain structure. Then, the void formation and failure lifetime of electromigration were simulated using the atomic density integral method based on the FEA analysis. The effect of grain orientation on current density and electromigration failure lifetime were also investigated. Based on the experiment and simulation results, we are able to draw conclusions about the effect of grain structure on the electromigration failure in lead-free solder bump. 


\section{ELECTROMIGRATION ACCELERATION TESTS}

\subsection{TEST SAMPLE}

Accelerated electromigration tests are often used to study electromigration failure. The electromigration lifetime at normal device operating conditions is usually more than a few years. In order to get results from electromigration-induced failure in a reasonable amount of time, accelerated electromigration tests are carried out at much higher current densities and elevated temperatures. The resistance of interconnects or solder bumps is monitored during the electromigration tests, and usually failure is determined at a certain criterion of resistance increase.

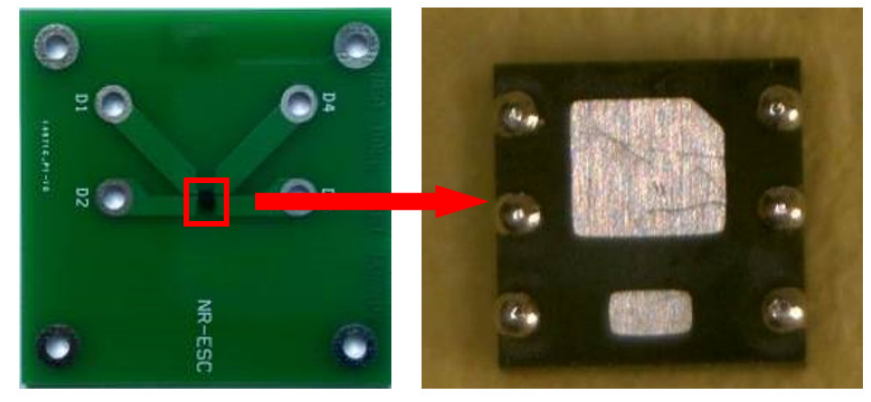

Fig. 1 Test sample of the Ball Grid Array

In this paper, the test sample of Ball Grid Array (BGA) device was used for the electromigration acceleration tests, which is shown in Figure 1 . The dimension of BGA device is $50 \mathrm{~mm} \times 50 \mathrm{~mm}$, which includes of solder bump, chip and PCB. The BGA device has 6 solder bumps with a diameter of $300 \mu \mathrm{m}$. Two solder bumps in the middle of BGA device were used to implement accelerated electromigration tests, and the role of other solder bumps is mainly in supporting chip. Copper $(\mathrm{Cu})$ layer was adopted to connect the chip device and solder bumps, shown as in Figure 2. This structure was designed so that electromigration tests are run in a two-bump

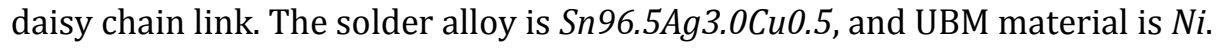

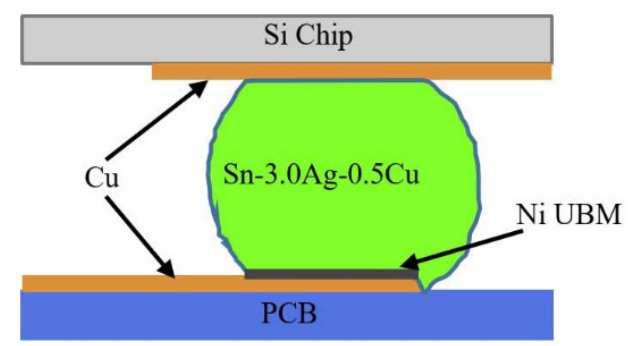

Fig. 2 Cross section of solder bump

\subsection{EXPERIMENTAL METHOD}

In accelerated electromigration tests, the BGA samples were connected with the power and data collection system. A thermocouple was pasted at the top surface of the chip and lower surface of the PCB, and then samples were placed into a thermostat for electromigration acceleration tests at constant current density and temperature. Solder bumps were subjected to the accelerated electromigration tests at $120^{\circ} \mathrm{C}$ with a current density of $1.4 \times 10^{4} \mathrm{~A} / \mathrm{cm}^{2}$. A 
$10 \%$ increase in resistance is used as a failure criterion and time of failure is recorded. EBSD tests were then further performed on bumps in untested samples (initial state) and samples that survived the accelerated electromigration tests. The complete information of the grain structure and grain orientations of each individual grain in solder bump can be obtained from the EBSD map. The experimental results will be analyzed to establish the relationship between grain structure and the electromigration failure mode of the solder bump.

\subsection{EXPERIMENTAL RESULTS}

Table 1 lists test conditions and results for all solder samples. Solder bumps 1\#-6\# are untested samples and Solder bumps 7\#-14\# those having survived the accelerated electromigration tests. It should be noticed that we can only get the time-to-failure (TTF) of the whole test sample but not of solder bump because two solder bumps of one test sample were used in accelerated electromigration tests.

Table 1 Test conditions and results for all samples

\begin{tabular}{|c|c|c|c|c|c|}
\hline Sample & Solder bump & $\begin{array}{c}\text { Current density } \\
\left(\mathrm{A} / \mathrm{cm}^{2}\right)\end{array}$ & $\begin{array}{l}\text { Numbers of } \\
\text { grain }\end{array}$ & Failure modes & $\begin{array}{l}\text { TTF } \\
\text { (h) }\end{array}$ \\
\hline \multirow{2}{*}{1} & $1 \#$ & - & 1 & - & \multirow{2}{*}{-} \\
\hline & $2 \#$ & - & 1 & - & \\
\hline \multirow{2}{*}{2} & 3\# & - & 1 & - & \multirow{2}{*}{-} \\
\hline & $4 \#$ & - & 2 & - & \\
\hline \multirow{2}{*}{3} & $5 \#$ & - & 2 & - & \multirow{2}{*}{ - } \\
\hline & $6 \#$ & - & $>5$ & - & \\
\hline \multirow[b]{2}{*}{4} & $7 \#$ & $1.4 \times 10^{4}$ & 1 & Voids occur at the cathode & \multirow[b]{2}{*}{56} \\
\hline & $8 \#$ & $1.4 \times 10^{4}$ & 1 & $\begin{array}{l}\text { Intermetallic compounds (IMC) form } \\
\text { between the UBM and solder bump }\end{array}$ & \\
\hline \multirow[t]{2}{*}{5} & 9\# & $1.4 \times 10^{4}$ & 1 & $\begin{array}{l}\text { Intermetallic compounds (IMC) form } \\
\text { between the UBM and solder bump }\end{array}$ & \multirow[t]{2}{*}{78.5} \\
\hline & $10 \#$ & $1.4 \times 10^{4}$ & $>5$ & Voids occur at the cathode & \\
\hline \multirow{2}{*}{6} & $11 \#$ & $1.4 \times 10^{4}$ & 1 & Ruptures Cracks occur at the cathode & \multirow{2}{*}{43.5} \\
\hline & $12 \#$ & $1.4 \times 10^{4}$ & $>5$ & Voids occur at the cathode & \\
\hline \multirow{2}{*}{7} & 13\# & $1.4 \times 10^{4}$ & $>5$ & Voids occur at the cathode & \multirow{2}{*}{86.6} \\
\hline & $14 \#$ & $1.4 \times 10^{4}$ & $>5$ & Ruptures Cracks occur at the cathode & \\
\hline
\end{tabular}

Figure 3 and Figure 4 show the EBSD images of some of the bumps in the untested sample. The images show that the tin-based solder bumps are comprised of very limited large grains, varying from single grain (seen as in Figure 3) and double grains structure (seen as in Figure 4). For the solder bumps with single grain structure, the direction of the c-axis of the $\beta$-Sn grains is relatively random. The angle between the c-axis of the $\beta$-Sn grains and the direction of the electric current is $10^{\circ}$ (Figure $3(\mathrm{a})$ ) and $90^{\circ}$ (Figure 3 (b)), respectively. But numerous small grains were found at the interface between the pad and solder bump. And most of the grain boundary angles are small below $10^{\circ}$. For the solder bumps with double grains structure, the misorientation angle of two grains is close to $60^{\circ}$. It is reported that a constant misorientation angle of $60^{\circ}$ is formed in the double grains structure of tin based solder bump, which was experimentally validated [5-6]. 


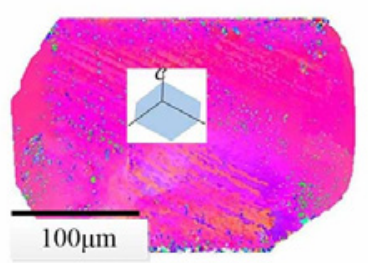

(a) 1 \# solder bump

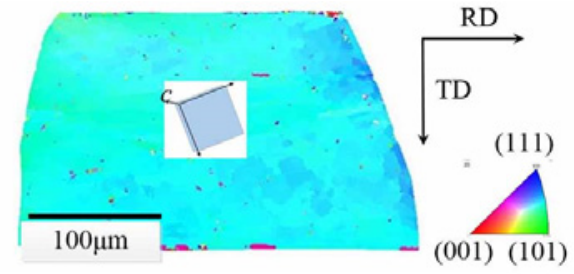

(b) 2\# solder bump

Fig. 3 EBSD images of solder bump with single grain structure
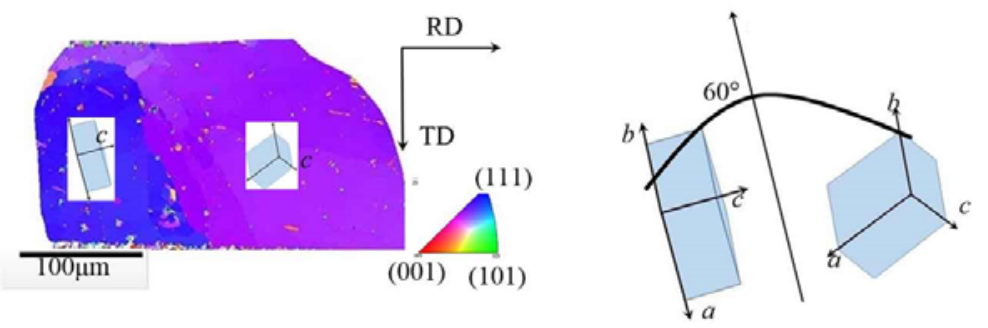

Fig. 4 EBSD image of solder bump with double grains structure for 5\# solder bump

Figures 5-7 show the SEM and EBSD images of some solder bumps with single grain structure that have been submitted to electromigration test. According to the experimental results, it was found that the failure mode of the solder bump with single grain structure is closely related to the angle $\theta$ between the $c$-axis of $\beta$-Sn grain and the electron flow direction. The angle $\theta$ is about $90^{\circ}, 15^{\circ}$ and $75^{\circ}$ in Figure 5, Figure 6 and Figure 7, respectively. It can be found that the void and rupture propagation is generated at the cathode, when $\theta$ is relatively large. However, $\mathrm{Cu}$ and $\mathrm{Ni}$ atoms in the substrate are diffused to the anode of solder bump and separated out at the anode, when $\theta$ is relatively small. In addition, the test samples in Figures 5-7 failed at very different times. The bump shown in Figure 5 has a TTF of $56 \mathrm{~h}$ at $10 \%$ resistance increase, the sample in Figure 6 has a TTF of 78.5h and the sample in Figure 5(c) has a TTF of 43.5h. The difference in TTF of the three samples is possible due to the difference in the microstructures. In next section, corresponding simulations will be done to predict this result. Figure 8 shows the SEM and EBSD image of solder bump with multiple grains structure that have gone through electromigration test. It can be seen that rupture appeared at the cathode, and some intermetallic compound (IMC) are generated at location A and B in the solder bump.
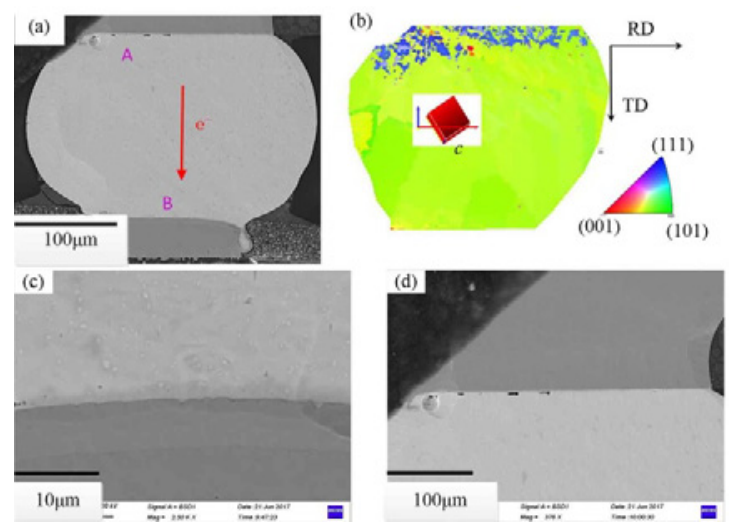

Fig. 5 SEM and EBSD images of $7 \#$ solder bump with single grain structure after electromigration test 

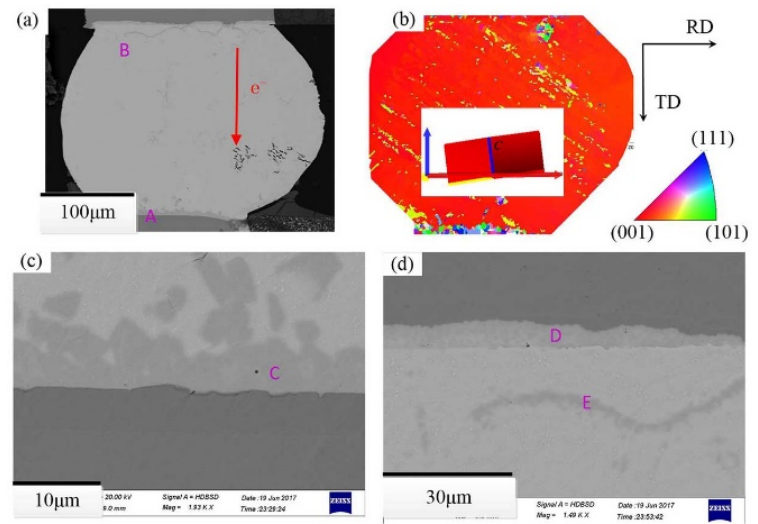

Fig. 6 SEM and EBSD images of 9\# solder bump with single grain structure after electromigration test
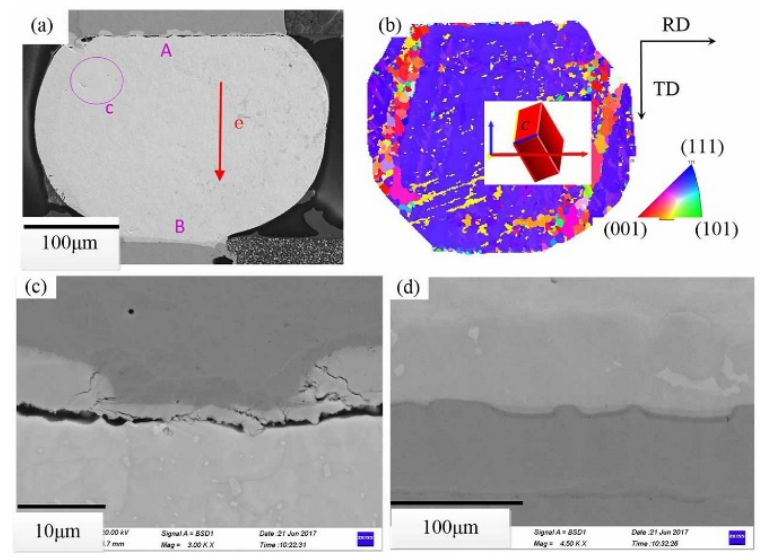

Fig. 7 SEM and EBSD images of 11\# solder bump with single grain structure after electromigration test
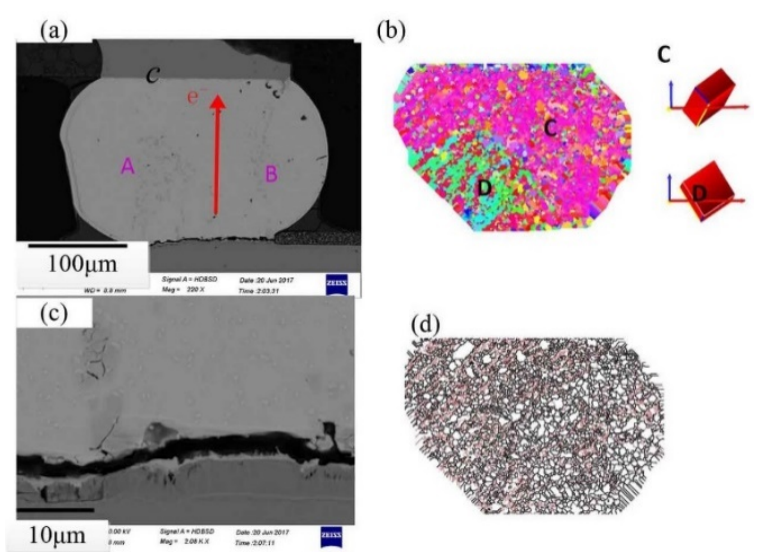

Fig. 8 SEM and EBSD images of 14\# solder bump with multiple grain structure after electromigration test 


\section{NUMERICAL MODELING OF ELECTROMIGRATION}

The variations in the failure mode of different grain structure have been found with the electromigration acceleration tests. To investigate further the effect of grain orientation on the electromigration failure of solder bump, the electromigration simulation of the solder bump with different grain orientation is performed based on the atomic density integral method.

\subsection{ATOMIC DENSITY INTEGRAL METHOD}

Electromigration is a diffusion process which is controlled by the mass continuity equation. The time dependent evolution equation of the local atomic density caused by the applied current is the mass balance (continuity) equation:

$$
\nabla \cdot \boldsymbol{q}+\frac{\partial c}{\partial t}=0
$$

where $c$ is the normalized atomic density, $c=C / C_{0}, C$ is the actual atomic density and $C_{0}$ is the initial (equilibrium state) atomic density in the absence of a stress field, $t$ is the time, $\boldsymbol{q}$ is the total normalized atomic flux.

Usually, the electromigration formulation of solder bump is driven by the effects of the electron wind force, stress gradients, temperature gradients, and the atomic density gradient. Thus:

$$
\boldsymbol{q}=\boldsymbol{q}_{E w}+\boldsymbol{q}_{T h}+\boldsymbol{q}_{S}+\boldsymbol{q}_{C}=\frac{c \boldsymbol{D}}{k_{B} T} e \boldsymbol{Z}^{*} \boldsymbol{j} \boldsymbol{\rho}-\frac{c \boldsymbol{D}}{k_{B} T} Q^{*} \frac{\nabla T}{T}-\frac{c \boldsymbol{D}}{k_{B} T} \Omega \nabla \sigma_{H}-\boldsymbol{D} \nabla c=c \cdot \boldsymbol{F}\left(T, \sigma_{H}, \boldsymbol{j}, \cdots\right)-\boldsymbol{D} \nabla c
$$

where $k$ is Boltzmann's constant, $e$ is the electronic charge, $Z^{*}$ is the effective charge tensor, $T$ is the absolute temperature, $\boldsymbol{\rho}$ is the resistivity tensor, $\boldsymbol{j}$ is the current density vector, $Q^{*}$ is the heat of transport, $\Omega$ is the atomic volume, $\sigma_{H}$ is the local hydrostatic stress, $\boldsymbol{D}$ is diffusivity tensor.

Because $\beta$-Sn has a BCT crystal structure, the diffusivity, electrical resistivity and effective charge number show an anisotropic character (different along $a$ and $c$ crystal directions). Let the $z$ axis be aligned with the crystal $c$ axis, so the only non-zero components in these tensors are $\hat{D}_{x x}=\hat{D}_{y y}=D_{a}, \hat{D}_{z z}=D_{c}, \hat{\rho}_{x x}=\hat{\rho}_{y y}=\rho_{a}, \hat{\rho}_{z z}=\rho_{c}, \hat{Z}_{x x}^{*}=\hat{Z}_{y y}^{*}=\hat{Z}_{a}^{*}, \hat{Z}_{z z}^{*}=Z_{c}^{*}$. We express the tensor components with respect to the global frame by simply rotating the tensors from the lattice to the global frame, which can be expressed as $D=R \hat{D} R^{T}, \rho=R \hat{\rho} R^{T}, Z^{*}=R \hat{Z}^{*} R^{T}$, where $R$ is the rotation matrix transforming components from the lattice to the global frame. Euler angles are normally used to describe the rotations of coordinates. The three rotation matrices of the Bunge Euler angles $\left(\varphi_{1}, \phi_{1} \varphi_{2}\right)$ are defined by Eqs. (3-5). And the rotation matrix can be expressed as $R=R_{3} R_{2} R_{1}$ :

$$
\begin{aligned}
& R_{1}\left(\varphi_{1}\right)=\left[\begin{array}{ccc}
\cos \left(\varphi_{1}\right) & \sin \left(\varphi_{1}\right) & 0 \\
-\sin \left(\varphi_{1}\right) & \cos \left(\varphi_{1}\right) & 0 \\
0 & 0 & 1
\end{array}\right] \\
& R_{2}(\Phi)=\left[\begin{array}{ccc}
1 & 0 & 0 \\
0 & \cos (\Phi) & -\sin (\Phi) \\
0 & -\sin (\Phi) & -\cos (\Phi)
\end{array}\right]
\end{aligned}
$$




$$
R_{3}\left(\varphi_{2}\right)=\left[\begin{array}{ccc}
\cos \left(\varphi_{2}\right) & 0 & -\sin \left(\varphi_{2}\right) \\
0 & 1 & 0 \\
\sin \left(\varphi_{2}\right) & 0 & \cos \left(\varphi_{2}\right)
\end{array}\right]
$$

The temperature dependence of the electrical resistivity along the $a$ and $c$ directions is treated as linear with:

$$
\rho_{q}=\rho_{q 0}\left[1+\alpha_{q}\left(T-T_{0}\right)\right]
$$

where $q$ is either $a$ or $c, \rho_{q 0}$ is electrical resistivity at the reference temperature $T_{0}=20^{\circ} \mathrm{C}$, and $\alpha_{q}$ represents the linear resistivity temperature coefficient.

The diffusivity tensor is also temperature dependent, where the diffusivities along the $a$ and $c$ direction can be expressed as:

$$
D_{q}=D_{q 0} \exp \left(-\frac{Q_{q}}{k T}\right)
$$

where $D_{q 0}$ is the intrinsic diffusivity and $Q_{q}$ is the activation energy for diffusion along the $a$ and $c$ direction.

For the EM evolution equation (Eq. (1)) on any enclosed domain $V$ with the corresponding boundary $\Gamma$, the atomic flux boundary conditions of solder bump can be expressed as:

$$
\boldsymbol{q} \cdot \boldsymbol{n}=q_{0} \quad \text { on } \quad \Gamma
$$

For blocking boundary condition:

$$
q_{0}=0 \quad \text { on } \Gamma
$$

At the initial time, the normalized atomic density is assumed to be:

$$
c_{0}=1
$$

In the finite element method, we seek an approximation solution for Eq. (1) to develop a simulation algorithm for the local atomic density in a solder bump. The first step is to multiply the time dependent EM evolution equation with a weighted residual function $w$ and integrate over the enclosed domain $V$ based on the vector identity by applying the Gauss-Ostrogradsky divergence theory to the product of the scalar function $w$ and the atomic flux vector field:

$$
\int_{V} w(\nabla \cdot \boldsymbol{q}+\dot{c}) d V=\int_{V} w \dot{c} d V+\int_{V} w \cdot \nabla \cdot \boldsymbol{q} d V=\int_{V} w \dot{c} d V-\int_{V} \frac{\partial w}{\partial \boldsymbol{n}} \cdot \boldsymbol{q} d V+\int_{\Gamma} w \cdot(\boldsymbol{q} \cdot n) d \Gamma=0
$$

From Eq. (11) with considering the atomic flux boundary condition of Eq. (5):

$$
\int_{V} w \dot{c} d V-\int_{V} \frac{\partial w}{\partial \boldsymbol{n}} \cdot\left\{c \cdot \boldsymbol{F}\left(T, \sigma_{m}, j, \cdots\right)-\boldsymbol{D} \nabla c\right\} d V=-\int_{\Gamma} w \cdot q_{0} d \Gamma
$$

Next, assume that $c=\sum_{j=1}^{n} \psi_{j} c^{j}, \dot{c}=\sum_{j=1}^{n} \psi_{j} \dot{c}^{j}$ and $w=\psi_{i}$ (for Galerkin Method), where $\psi_{i}$ is the shape function of the element. After element discretization, the matrix form of Eq. (12) can be written as:

$$
[\boldsymbol{M}]\{\dot{c}\}+[\boldsymbol{K}]\{c\}=\{\boldsymbol{Y}\}
$$


if the mass matrix $[\boldsymbol{M}]$ is independent of time, the stiffness matrix $[\boldsymbol{K}]$ will remain constant in an incremental step, where we consider the current density $\boldsymbol{j}$ and the local hydrostatic stress $\sigma_{m}$ are not varied in the current incremental step, $\{\boldsymbol{Y}\}$ is the known term.

The most commonly used local iteration scheme for solving the above Eq. (11) is the $\alpha$-family of approximation method. Thus, Eq. (13) can be written as:

$$
([\boldsymbol{M}]+\alpha \Delta t[\boldsymbol{K}])\left\{c_{t_{i+1}}\right\}=([\boldsymbol{M}]-(1-\alpha) \Delta t[\boldsymbol{K}])\left\{c_{t_{i}}\right\}+\{\overline{\boldsymbol{Y}}\}_{i, i+1}
$$

where:

$$
\{\overline{\boldsymbol{Y}}\}_{i, i+1}=(1-\alpha) \Delta t\{\overline{\boldsymbol{Y}}\}_{t_{i}}+\alpha \Delta t[\boldsymbol{M}]\{\overline{\boldsymbol{Y}}\}_{t_{i+1}}
$$

Thus, the normalized atomic density $c$ in the $(i+1)^{\text {th }}$ step can be obtained based on Eq. (13) in terms of the corresponding value in the $i^{t h}$ step. Since the initial atomic density $c_{0}=1$ is known, the above equations provide the solution to $c$ at any time step. Specifically, the initial distributions of the current density, temperature and stress in the solder bump are obtained by the 3D finite element method analysis based on ANSYS software. Then, atom density distribution in the solder bump is solved based on the ADI algorithm using the user-defined FORTRAN code. A link between the ANSYS and the FORTRAN codes is also developed based on ANSYS program design language (APDL) to transfer the data.

Assume that there is a critical atomic density for void initiation $c_{\text {min }}^{*}$. When the average atomic density value of the elements is less than or equal to $c_{\min }^{*}$, the void will appear. The values of $c_{\text {min }}^{*}$ can be obtained from experiments. The criterion we cited here is based on test data, for solder alloys $c_{\text {min }}^{*}=0.85$. For a solder bump failure criterion, a $10 \%$ increment in electrical resistance of the bump is considered EM failure, which is consistent with the accelerated electromigration test. This criterion is used in this paper to obtain the final TTF.

\subsection{THE FINITE ELEMENT MODEL}

The finite element model was built according to the electromigration acceleration test of BGA sample in ANSYS finite element software, and the boundary conditions were set to be identical with the experimental working conditions, as shown in Figure 9. The domain is meshed with hexahedral elements. The anisotropic thermal, electrical and diffusion properties of pure $\beta$-Sn are used here for the lead-free solder bump, as shown in Table 2 [19-22]. Other materials are isotropic. Their related material properties used in the simulation are taken from references [13-14, 17].

At first, the solder bumps are assumed to be single crystal. To understand the effect of grain orientation on the electromigration failure, the thermoelectric-structural simulation is repeated for different grain orientations of the single crystal bumps. Bunge Euler angles $\left(\varphi_{1}, \phi\right.$, $\varphi_{2}$ ) are used to represent grain orientation. The first is about rotation the $Z$-axis with an angle $\varphi_{1}$, the second about the $X$-axis with an angle $\phi$, and the third again about the $Z$-axis with an angle $\varphi_{2}$. Due to the symmetry of $S n$ crystal structure, the third angle $\varphi_{2}$ is set to zero and the first two angles $\varphi_{1}$ and $\phi$ are increased from $0^{\circ}$ to $180^{\circ}$ at $15^{\circ}$ intervals. 


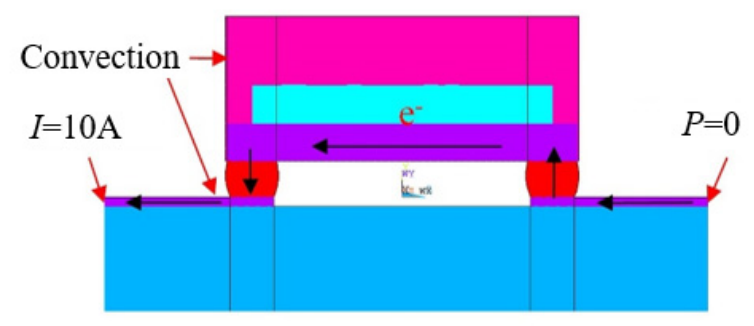

Fig. 9 Schematic diagram of boundary conditions

Table 2 Anisotropic thermal, electrical and diffusion properties of $\beta$-Sn

\begin{tabular}{|c|c|c|}
\hline Material property & a-axis & c-axis \\
\hline$\rho_{q 0}(\Omega \cdot \mathrm{m})$ & $14.3 \times 10^{-8}$ & $9.9 \times 10^{-8}$ \\
\hline$\alpha_{q}(/)$ & 0.00447 & 0.00469 \\
\hline$Z^{*}$ & -10 & -16 \\
\hline$D_{q 0}\left(\mathrm{~m}^{2} / \mathrm{s}\right)$ & 0.00128 & 0.0021 \\
\hline$Q_{q}(\mathrm{~kJ} / \mathrm{mol})$ & 109.0 & 108.4 \\
\hline
\end{tabular}

To better understand electromigration failure of double grains solder bump, two different bicrystal configurations are considered for the solder bumps in Figure 10. The bi-crystals have a vertical grain boundary (perpendicular to the substrate) through the centre of the grain oriented either parallel to the entering current direction (Figure 10(a)) or perpendicular to the entering current direction (Figure 10(b)). In each case, one grain is oriented with the $c$-axis vertical (almost parallel to the current direction) and the other with the rotation of $60^{\circ}$ by $a$ axis or $b$-axis.

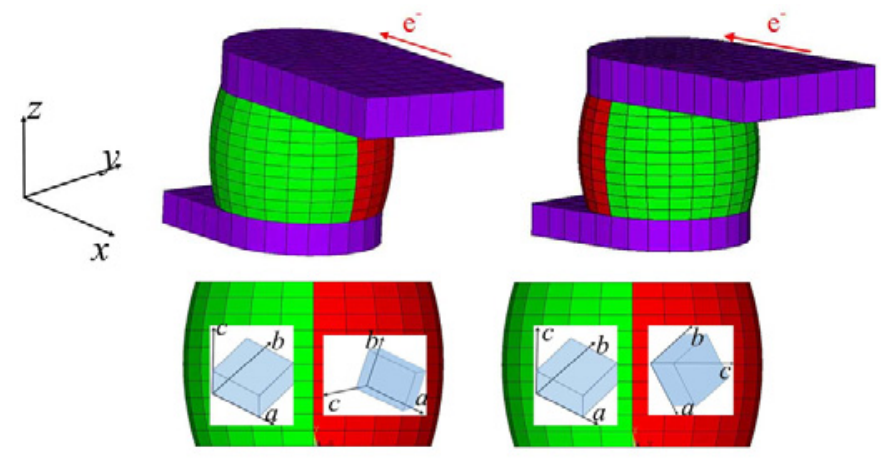

Fig. 10 Finite element model of bi-crystal solder bump

\subsection{RESULTS ANALYSIS}

Firstly, the single crystal model was simulated. Figure 11 shows the current density distribution of the solder bump when the $a, b, c$ axes of $S n$ crystal are aligned with the global $x$, $y$ and $z$ directions (Bunge Euler angles $\left[\begin{array}{lll}0 & 0 & 0\end{array}\right]$ ). The maximum magnitude of current density is $2.53 \times 10^{4} \mathrm{~A} / \mathrm{cm}^{2}$ at the bottom of solder bump where electrons enter the bump from the $\mathrm{Cu}$ trace. The current density at top of solder bump is about $2.30 \times 10^{4} \mathrm{~A} / \mathrm{cm}^{2}$, which is also relatively high. The distribution of the current density with different grain orientation is nearly the same, but the maximum current density of solder bump is obviously different. We found 
that the maximum current density is mainly determined by the Euler angle $\Phi$, the peak current density is obtained when $\Phi=90^{\circ}$.

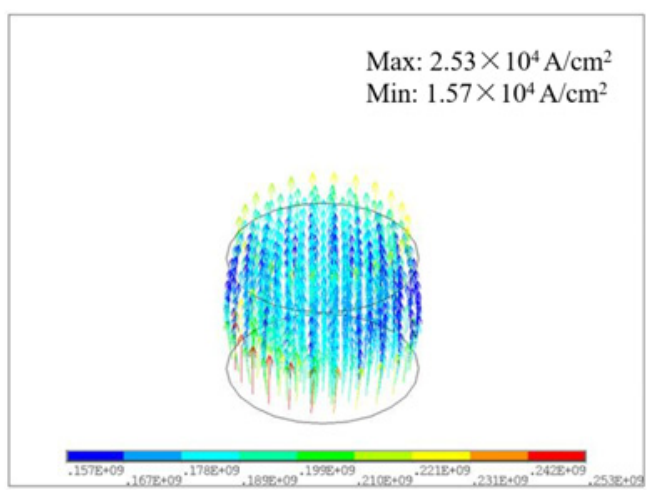

Fig. 11 Current density distribution of single crystal solder bump at grain orientation [0 0 0]

As mentioned before, the atomic density indicates the level of material depletion at a given location in the bump and thus is a very important factor in determining electromigrationinduced failure. Figure 12 shows the atomic density distribution of solder bump at grain orientation $\left[\begin{array}{lll}0 & 0 & 0\end{array}\right]$. As expected, the minimum value of the atomic density appears at the top right corner of the solder bump, where electrons enter the bump from the $\mathrm{Cu}$ trace. The electromigration failure could easily occurre at the region with the lowest atomic density, which is consistent with the location of ruptures observed in the electromigration acceleration test.

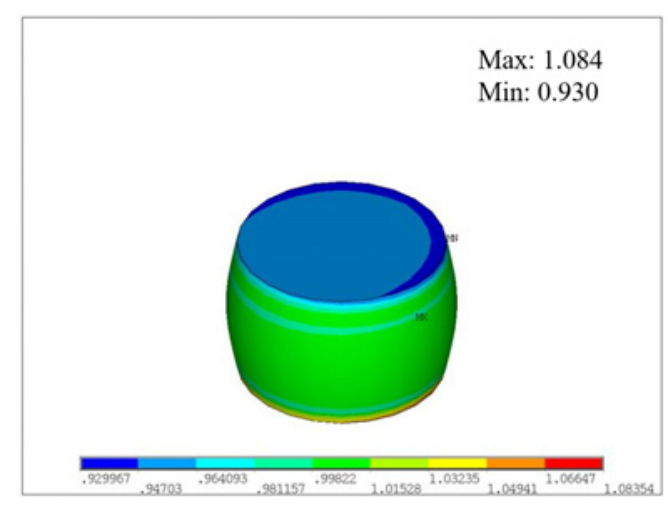

Fig. 12 Atomic density distribution of single crystal solder bump at grain orientation [0 0 0]

The TTF of single crystal solder bump with different grain orientation was calculated using atomic density integral method, as shown in Figure 13. It can be found that $\Phi$ has significant effect on the TTF of the single grain solder bump. The minimum lifetime of the solder bump is

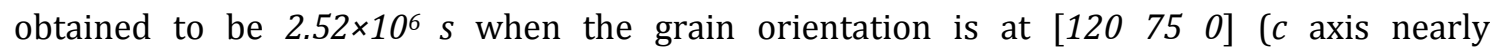
perpendicular to the current direction). When $\Phi=0^{\circ}$ or $180^{\circ}(\mathrm{c}$ - axis is almost aligned with the current direction), the lifetime of solder bump reaches its maximum of $3.735 \times 10^{6} \mathrm{~s}$, which is 1.5 times higher than the minimum electromigration lifetime. It is consistent within the TTF results in the electromigration acceleration test. This could be attributed to higher diffusion velocity of $\beta$-Sn along $a, b$ axis (1.8 times) than that of along the $c$ axis. Lu et al. [9] also found that electromigration failure occurs fast when $c$ axis is strongly misaligned in relation to the current direction. 


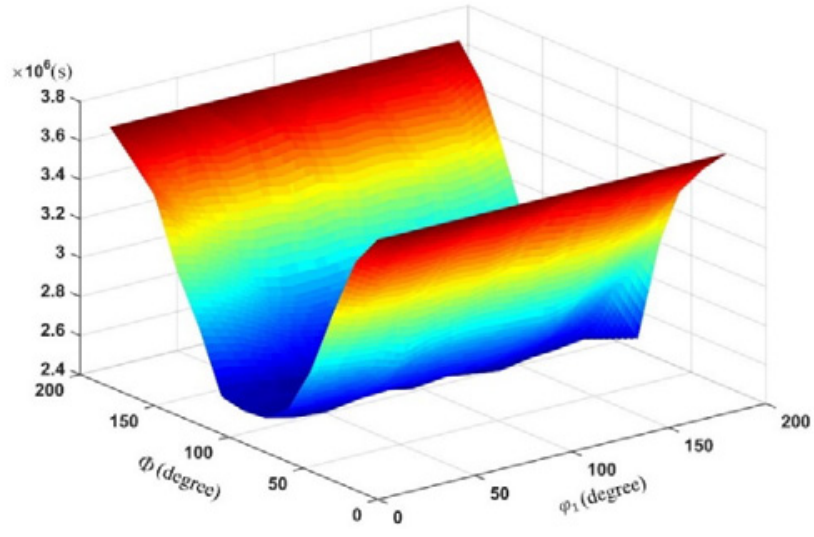

Fig. 13 TTF of the single crystal solder bump with different grain orientation

In order to study the electromigration failure of double grain solder bump, a twin grain model misorientation of with $60^{\circ}$ (as shown in Figure 10) was built and performed by ADI method. Figure 14 and Figure 15 show the atomic density distribution of double grains solder bump. It is found that the location of the grain boundary has certain effect on the atomic density distribution. The peak atomic density in the double grain solder bump with grain boundary vertical to current direction is smaller than in one parallel to current direction.

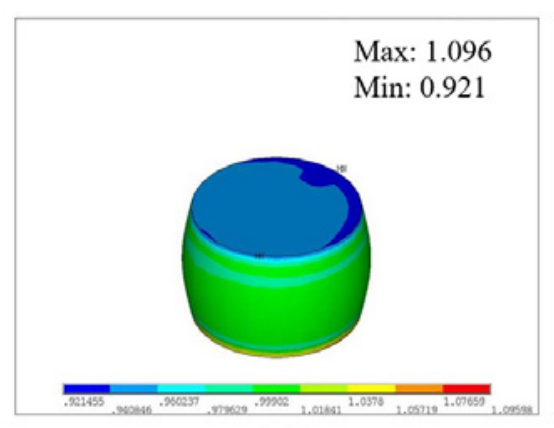

(a)

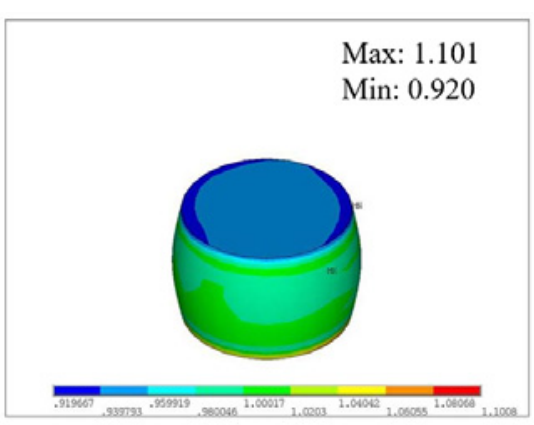

(b)

Fig. 14 Atomic density distribution of double grains solder bump when current is parallel to the grain boundary

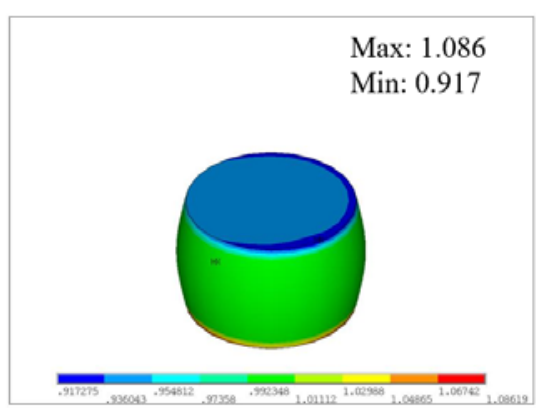

(a)

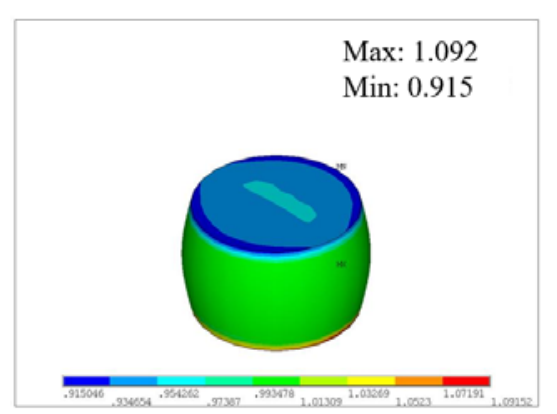

(b)

Fig. 15 Atomic density distribution of double grains solder bump when current is vertical to the grain boundary 


\section{CONCLUSIONS}

The electromigration acceleration test and finite element analysis have been implemented in studying the effect of microstructure the electromigration failure in lead-free solder bump. The failure mode of single grain solder bump is closely related to the angle $\theta$ between the $c$-axis of $\beta$-Sn grain and the electron flow direction. The void and rupture propagation generated at the cathode when $\theta$ is relatively large, while $\mathrm{Cu}$ and $\mathrm{Ni}$ atoms in the substrate are diffused to the anode of solder bump and separated out at the anode when $\theta$ is relatively small. The orientation angle $\Phi$ that specifies the rotation of the $c$ axis, has significant effect on the electromigration failure lifetime of the single grain solder bump. It could lead to the longest electromigration failure lifetime when the crystal $c$ axis is nearly aligned with the current direction. In the double grains solder bump studies, the position of grain boundary has certain effect on the electromigration failure. The double grain solder bump with grain boundary parallel to current direction has less longer TTF.

\section{ACKNOWLEDGMENTS}

This work is supported by National Science Foundation of China under Grant Nos. 51605252, 5187052611 and 51501100.

\section{REFERENCE}

[1] K.N. Tu, Reliability Challenges in 3D IC Packaging Technology, Microelectronics Reliability, Vol. 51, No. 3, pp. 517-523, 2011.

https://doi.org/10.1016/j.microrel.2010.09.031

[2] C. Ho, P. Lee, C. Chen and C. Yang, Electromigration in 3D-IC Scale $\mathrm{Cu} / \mathrm{Sn} / \mathrm{Cu}$ Solder Joints, Journal of Alloys and Compounds, Vol. 676, pp. 361-368, 2016.

https://doi.org/10.1016/j.jallcom.2016.03.134

[3] J. Ni, Modelling Microstructure Effects on Electromigration in Tin-Based Solder Bumps. Troy: Rensselaer Polytechnic Institute, 2017.

[4] S. Wei, H.C. Ma, J.Q. Chen and J.D. Guo, Extreme Anisotropy of Electromigration: Nickel in Single-Crystal Tin, Journal of Alloys and Compounds, Vol. 687, pp. 999-1003, 2016.

https://doi.org/10.1016/j.jallcom.2016.06.253

[5] M.L. Huang, J.F. Zhao, Z.J. Zhang and N. Zhao, Dominant Effect of High Anisotropy in $\beta$-Sn Grain On Electromigration-Induced Failure Mechanism in Sn-3.0Ag-0.5Cu Interconnect, Journal of Alloys and Compounds, Vol. 678, pp. 370-374, 2016.

https://doi.org/10.1016/j.jallcom.2016.04.024

[6] M.L. Huang, J.F. Zhao, Z.J. Zhang and N. Zhao, Role of Diffusion Anisotropy in $\beta$-Sn in Microstructural Evolution of Sn-3.0Ag-0.5Cu Flip Chip Bumps Undergoing Electromigration, Acta Materialia, Vol. 100, pp. 98-106, 2015.

https://doi.org/10.1016/j.actamat.2015.08.037 
[7] A.M. Maniatty, J. Ni, Y. Liu and H. Zhang, Effect of Microstructure on ElectromigrationInduced Stress, Journal of Applied Mechanics, Transactions ASME, Vol. 83, No. 1, pp. 11010, 2016.

[8] Y. Wang and K.H. Lu, Effects of Sn Grain Structure on the Electromigration of Sn-Ag Solder Joints, Journal of Material Research, Vol. 27, No. 8, pp. 1131-1141, 2012. https://doi.org/10.1557/jmr.2012.10

[9] M. Lu, D. Shih, P. Lauro, C. Goldsmith and D.W. Henderson, Effect of Sn Grain Orientation On Electromigration Degradation Mechanism in High Sn-based Pb-free Solders, Applied Physics Letters, Vol. 92, No. 21, pp. 211909, 2008. https://doi.org/10.1063/1.2936996

[10] K. Lee, K.-S. Kim, Y. Tsukada, K. Suganuma, K. Yamanaka, S. Kuritani and M. Ueshima, Influence of Crystallographic Orientation of Sn-Ag-Cu on Electromigration in Flip-Chip Joint, Microelectronics Reliability, Vol. 51, No. 12, pp. 2290-2297, 2011.

https://doi.org/10.1016/j.microrel.2011.05.003

[11] C. Basaran and M. Lin, Electromigration Induced Strain Field Simulations for Nanoelectronics Lead-Free Solder Joints, International Journal of Solids and Structures, Vol. 44, No. 14-15, pp. 4909-4924, 2007. https://doi.org/10.1016/j.ijsolstr.2006.12.011

[12] F. He and C.M. Tan, Circuit Level Interconnect Reliability Study Using 3D Circuit Model, Microelectronics Reliability, Vol. 50, No. 3, pp. 376-390, 2010.

https://doi.org/10.1016/j.microrel.2009.12.009

[13] Y. Liu, Y. Zhang and L. Liang, Prediction of Electromigration Induced Voids and Time to Failure for Solder Joint of a Wafer Level Chip Scale Package, IEEE Transactions on Components and Packaging Technologies, Vol. 33, No. 3, pp. 544-552, 2010.

https://doi.org/10.1109/TCAPT.2010.2042717

[14] L. Liang, Y. Zhang and Y. Liu, Prediction of Electromigration Failure of Solder Joints and its Sensitivity Analysis, Journal of Electronic Packaging, Vol. 133, No. 3, pp. 31002, 2011. https://doi.org/10.1115/1.4004658

[15] J. Ni, Y. Liu, J. Hao, A. Maniatty and B. Connell, Modeling Microstructure Effects on Electromigration in Lead-free Solder Joints, 64th Electronic Components and Technology Conference, pp. 1241-1246, 2014. https://doi.org/10.1109/ECTC.2014.6897450

[16] H. Ceric, R. Lacerda, J. Cervenka and S. Selberherr, A Comprehensive TCAD Approach for Assessing Electromigration Reliability of Modern Interconnects, IEEE Transactions Device Material. Reliability, Vol. 9, No. 1, pp. 9-19, 2009.

https://doi.org/10.1109/TDMR.2008.2000893

[17] Y.X. Zhang, L. Liang and Y. Liu, Investigation for Electromigration-Induced Hillock in a Wafer Level Interconnect Device, 60th Electronic Components and Technology Conference, pp. 617-624, 2010. https://doi.org/10.1109/ECTC.2010.5490862

[18] Y. Zhang, Electromigration Failure Prediction and Reliability Evaluation of Solder Bumps for FCBGA Package, Engineering Transactions, Vol. 2, No. 63, pp. 215-232, 2015.

[19] Y.S. Touloukian, R.W. Powell, C.Y. Ho and P.G. Klemens, Thermophysical Properties of Matter-The TPRC Data Series. Volume 1. Thermal Conductivity-Metallic Elements and Alloys, New York: Plenum, 1970. https://doi.org/10.1007/978-1-4615-9600-4 
[20] K.J. Puttlitz and K.A. Stalter, Handbook of Lead-Free Solder Technology for Microelectronic Assemblies, New York: Marcel Dekker, 2004. https://doi.org/10.1201/9780203021484

[21] H.B. Huntington, Effect of Driving Forces on Atom Motion, Thin Solid Films, Vol. 25, pp. 265-280, 1975. https://doi.org/10.1016/0040-6090(75)90047-4

[22] F.H. Huang and H. B. Huntington, Diffusion of Sb124, Cd109, Sn113 and Zn65 in Tin, Physics Review B, Vol. 9, No. 4, pp. 1479-1488, 1974.

https://doi.org/10.1103/PhysRevB.9.1479 